\title{
Erratum to: Identification of QTLs for seed and pod traits in soybean and analysis for additive effects and epistatic effects of QTLs among multiple environments
}

Zhe Yang $\cdot$ Dawei Xin · Chunyan Liu

Hongwei Jiang • Xue Han · Yanan Sun •

Zhaoming Qi · Guohua Hu • Qingshan Chen

Published online: 20 November 2013

(C) Springer-Verlag Berlin Heidelberg 2013

\section{Erratum to: Mol Genet Genomics}

DOI 10.1007/s00438-013-0779-z

In the original publication, inadvertantly the corresponding authorship was given to Dr. Zhe Yang. The correct corresponding authors of this article are Dr. Guohua $\mathrm{Hu}$ and Dr. Qingshan Chen.

The online version of the original article can be found under doi:10.1007/s00438-013-0779-z.

Z. Yang $\cdot$ D. Xin $\cdot$ Y. Sun $\cdot$ Z. Qi $\cdot$ Q. Chen $(\bowtie)$

Key Laboratory of Soybean Biology in Chinese Ministry

of Education (Key Laboratory of Soybean Biology and Breeding/

Genetics of Chinese Agriculture Ministry), Northeast Agricultural

University, Harbin 150030, China

e-mail: qshchen@126.com

Z. Yang

e-mail: yz78100@163.com

Z. Yang $\cdot$ H. Jiang $\cdot X$. Han

Agronomy College, Northeast Agriculture University,

Harbin 150030, China

Z. Yang

Soybean Research Institute, Heilongjiang Academy

of Agricultural Sciences, Harbin 150086, China

C. Liu $\cdot$ G. Hu $(\bowtie)$

The Crop Research and Breeding Center of Land-Reclamation,

Harbin 150090, China

e-mail: hugh757@vip.163.com 